\title{
Expression of human telomerase reverse transcriptase mediates the senescence of mesenchymal stem cells through the PI3K/AKT signaling pathway
}

\author{
QIANG ZHAO $^{1,2^{*}}$, XUE-YAO WANG $^{3 *}$, XIAO-XIA YU $^{1}$, YING-XIAN ZHAI $^{1}$, \\ $\mathrm{XU} \mathrm{HE}^{1}, \mathrm{SHAN} \mathrm{WU}^{1}$ and YING-AI SHI ${ }^{1}$ \\ ${ }^{1}$ Key Laboratory of Pathobiology, Ministry of Education, College of Basic Medical Sciences, Jilin University; \\ ${ }^{2}$ Department of Pediatric Surgery, The First Hospital, Jilin University; ${ }^{3}$ Norman Bethune College \\ of Medical Sciences, Jilin University, Changchun, Jilin 130021, P.R. China
}

Received February 28, 2015; Accepted July 8, 2015

DOI: $10.3892 / \mathrm{ijmm} .2015 .2284$

\begin{abstract}
Multipotent mesenchymal stem cells (MSCs) are widely used as seed cells in studies of tissue engineering and regenerative medicine; however, their clinical application is limited due to replicative senescence. It has been demonstrated that telomerase expression extends the lifespan and maintains the bone-forming ability of MSCs; however, the detailed role and the underlying molecular mechanisms in MSCs remain largely unknown. In the present study, we found that senescence was associated with human telomerase reverse transcriptase (hTERT) expression, and telomere length and telomerase activity. We established a short interfering RNA (siRNA) targeting hTERT and a gene expression vector carrying hTERT and transfected these into the MSCs to investigate the detailed role and the underlying molecular mechanisms of action of hTERT in MSCs. We found that the downregulation of hTERT by siRNA markedly decreased telomere length and telomerase activity in the MSCs, whereas the overexpression of hTERT increased telomere length and telomerase activity in the MSCs. The downregulation of hTERT inhibited cell proliferation and promoted the senescence and apoptosis of MSCs, whereas the upregulation of hTERT increased cell proliferation and decreased the senescence and apoptosis of MSCs. Of note, we also found that the activation of the PI3K/AKT signaling pathway was mediated by hTERT and that blocking this pathway using LY294002 inhibited hTERT expression, induced senescence and decreased the proliferation of MSCs. These
\end{abstract}

Correspondence to: Professor Ying-Ai Shi, Key Laboratory of Pathobiology, Ministry of Education, College of Basic Medical Sciences, Jilin University, 126 Xinmin Street, Chaoyang, Changchun, Jilin 130021, P.R. China

E-mail: shiyingai5312@126.com

*Contributed equally

Key words: mesenchymal stem cells, human telomerase reverse transcriptase, senescence, PI3K/AKT findings reveal a previously unknown regulatory mechanism of hTERT, indicating that hTERT mediates the senescence of MSCs through the PI3K/AKT signaling pathway.

\section{Introduction}

Mesenchymal stem cells (MSCs) are multipotent stem cells derived from the mesoderm, and possess potent proliferative potential, and the capacity for self-renewal and multilineage differentiation (1-3). MSCs grown in vitro are capable of differentiating into a number of cell types including osteoblasts, chondrocytes and adipocytes $(4,5)$. MSCs are of great interest to researchers for their potential applications in the field of regenerative medicine and tissue engineering (6). Prolonged passaging of the in vitro culture environment is a prerequisite for acquiring a suitable number of MSCs for use in cell therapy. The process may, however, lead to adverse effects on the physiological properties of MSCs, such as stemness, proliferation and differentiation potency (6). In addition, during long-term in vitro culture, MSCs easily develop cellular senescence, consequently further limiting the number of cell doublings (7). It is therefore important to understand the mechanisms of the senescence of MSCs to reverse or prevent the aging processes in these cells.

In normal somatic cells, each cell division is associated with the shortening of telomeric DNA at the end of each chromosome, which leads to the cessation of replication, and the eventual arrest of cell growth and proliferation (8-10). The activation of telomerase is responsible for extending telomere length at the end of chromosomes, which helps prevent telomere erosion and inhibit replicative senescence in vitro $(11,12)$. Human telomerase reverse transcriptase (hTERT) is a catalytic subunit of human telomerase $(13,14)$, which provides the reverse transcriptase activity needed to maintain the length of the telomere (15). It has been shown that overexpression of hTERT increases the cell lifespan of ameloblastoma cells (16), human fibroblasts (17), adipose-derived stem cells (18) and endothelial cells (19) in vitro. Several studies have demonstrated that the modification of human MSCs with the hTERT gene generates cells with an improved ability for proliferation and cell renewal that retain their potential to differentiate into osteocytes, adipocytes, 
chondrocytes and gingival epithelial cell lines (20-25). However, the detailed role and the underlying molecular mechanisms of action of hTERT in MSCs remain largely unknown. Therefore, in the present study, we examined the effects of hTERT expression on the proliferation, apoptosis and senescence of rat MSCs, as well as the underlying molecular mechanisms.

\section{Materials and methods}

Isolation and culture of MSCs. The MSCs used in the present study were obtained from 17- to 18-month old male Sprague Dawley rats (Tonghua Laboratory Animal Center, Beijing, China). The isolation and culture of the MSCs were performed as previously described $(26,27)$. In brief, the MSCs were isolated from the bone marrow of the femurs and tibias of SD rats by inserting a 21-gauge needle into the shaft of the bone and flushing it with $\alpha$-modified Eagle's medium ( $\alpha$-MEM; Invitrogen, Carlsbad, CA, USA) and cultured for 1-2 days. Non-adherent cells were then removed, and adherent cells representing MSCs were washed twice with phosphate-buffered saline (PBS; Sigma-Aldrich, St. Louis, MO, USA). The cells were then incubated for 7-10 days in DMEM medium supplemented with $10 \%$ fetal bovine serum (FBS; HyClone, Logan, UT, USA) to reach confluence and were extensively propagated for further experiments. The culture medium was replaced every 3-4 days and the cells were passaged at $70-80 \%$ confluence. The morphology of MSCs at passages 3, 7,9 and 12 was observed under an IX51 inverted microscope (Olympus Corp., Tokyo, Japan). MSCs at passage 3 were harvested and resuspended in culture medium at a density of $1 \times 10^{6} \mathrm{cells} / \mathrm{ml}$. The surface markers of MCS were examined using flow cytometry and 4 antibodies against rat surface antigens (CD29, CD45, CD71 and CD90; BD Biosciences, San Jose, CA, USA).

Senescence-associated $\beta$-galactosidase (SA- $\beta$-gal) staining. The cells $\left(4 \times 10^{4}\right)$ were seeded into 6 -well plates. After $48 \mathrm{~h}$, the cells were washed twice with PBS and fixed for $10 \mathrm{~min}$. After removing the fixative, the cells were washed twice with PBS and stained with the staining solution provided by the $\beta$-Galactosidase Reporter Gene Staining kit (Sigma-Aldrich) for $12 \mathrm{~h}$ at $37^{\circ} \mathrm{C}$, and the percentage of $\beta$-galactosidase-positive cells was then determined by randomly counting 5 fields on a phase contrast microscope (Olympus Corp.).

Reverse transcription-quantitative polymerase chain reaction (RT-qPCR). Total RNA was extracted from the MSCs using TRIzol reagent (Invitrogen). The RNA was reversetranscribed into cDNA using a PrimeScript ${ }^{\mathrm{TM}}$ RT Reagent kit according to the manufacturer's instructions (Takara Bio, Dalian, China). Quantitative PCR (qPCR) was carried out using SYBR-Green Real-Time PCR Master Mix (Toyobo Co., Ltd., Osaka, Japan) and qPCR amplification equipment. The PCR reactions were prepared in duplicate and heated to $95^{\circ} \mathrm{C}$ for 5 min followed by 40 cycles of denaturation at $95^{\circ} \mathrm{C}$ for $15 \mathrm{sec}$, annealing at $54^{\circ} \mathrm{C}$ for $30 \mathrm{sec}$, and extension at $72^{\circ} \mathrm{C}$ for $30 \mathrm{sec}$. Standard curves (cycle threshold values vs. template concentration) were prepared for each target gene and the endogenous reference (GAPDH) in each sample. The quantification of the samples was carried out using LightCycler software version 3.5 (Roche, Mannheim, Germany) with the $2^{-\triangle \Delta C T}$ method. The sequences of the hTERT and GAPDH primers were consistent with those of our previous study (28): hTERT forward, 5'-GGAGCAAGTTGCAAAGCATTG-3' and reverse, 5'-TCCCACGACGTAGTACATGTT-3'; GAPDH forward, 5'-TG TGGGCATCAATGGATTTGG-3' and reverse, 5'-ACACCATGTATTCCGGGTCAAT-3'.

Detection of telomerase activity and measurement of telomere length. Telomerase activity was determined using the telomeric repeat amplification protocol (TRAP) with the TeloTAGGG PCR enzyme-linked immunosorbent assay (ELISA) kit $\approx$ (Roche) according to the manufacturer's instructions.

Genomic DNA from the cultured cells was isolated using the High Pure PCR Template Preparation kit (Roche) and telomere length was estimated using the TeloTAGGG Telomere Length assay kit (Roche) as previously described (28).

Cell proliferation assay. The MSCs were seeded in a 96-well plate at a density of $1 \times 0^{4}$ cells/well. Cell proliferation was determined at the indicated time points using the Cell Counting kit-8 (CCK-8; Dojindo Laboratories, Kumamoto, Japan) according to the manufacturer's instructions. The absorbance was read using an ELISA plate reader (Thermo Labsystems, Vantaa, Finland) at $450 \mathrm{~nm}$.

Plasmid construction and transfection. The plasmid, pGCsilencer-siRNA-hTERT (pSi-hTERT), encoding siRNA specific to hTERT was constructed as previously described (28). The plasmid pCI-neo-hTERT (phTERT) containing the hTERT coding region was kindly provided by Professor Ximin Guo (Academy of Military Medical Sciences, Beijing, China). The plasmids, pSi-hTERT and phTERT, were transiently transfected into the MSCs at passage 9 (late passage) using Lipofectamine ${ }^{\mathrm{TM}} 2000$ reagent (Invitrogen) according to the manufacturer's instructions. The transfection efficiency was evaluated by RT-qPCR and western blot analysis following transfection with various plasmids. Untransfected cells were used as controls in this experiment and the following experiments.

Western blot analysis. The cells were harvested by centrifugation, and the cell pellet was resuspended in lysis buffer (Sigma-Aldrich) containing proteinase inhibitors and incubated on ice for $30 \mathrm{~min}$. Following centrifugation at $14,000 \mathrm{x} \mathrm{g}$ for $30 \mathrm{~min}$ at $4^{\circ} \mathrm{C}$, the supernatant containing total cell extract was collected, and the concentrations of total cellular protein were determined using the Bradford protein assay (Bio-Rad Laboratories, Marnes-la-Coquette, France). Equal amounts of protein $(20 \mu \mathrm{g})$ were separated by $10 \%$ gradient sodium dodecyl sulfate-polyacrylamide electrophoresis (SDS-PAGE) gels and transferred onto polyvinylidene fluoride (PVDF) membranes (Millipore, Billerica, CA, USA) using the Trans-Blot Turbo Transfer system (Bio-Rad Laboratories). The membranes were incubated in blocking buffer (TBST containing 5\% skim milk) for $1 \mathrm{~h}$ at room temperature to block non-specific protein binding and then incubated with the following primary antibodies overnight at $-4^{\circ} \mathrm{C}$ : anti-hTERT (1:2,000; Cat. no. Sc-7204; Santa Cruz Biotechnology, Inc., Santa Cruz, CA, USA), anti-GAPDH (1:5,000; Cat. no. 3683), anti-PI3K (1:2,000; Cat. no. 4249), anti-phosphorylated (p)-PI3K (Tyr458, 1:1,500; Cat. no. 4228), anti-AKT (1:1,000; Cat. no. 2920) and anti-p-AKT (Ser473; 
A
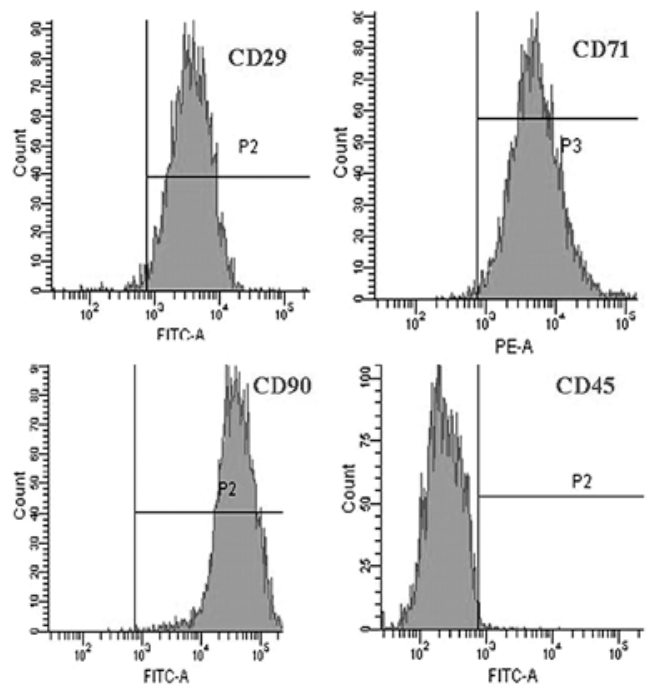

C

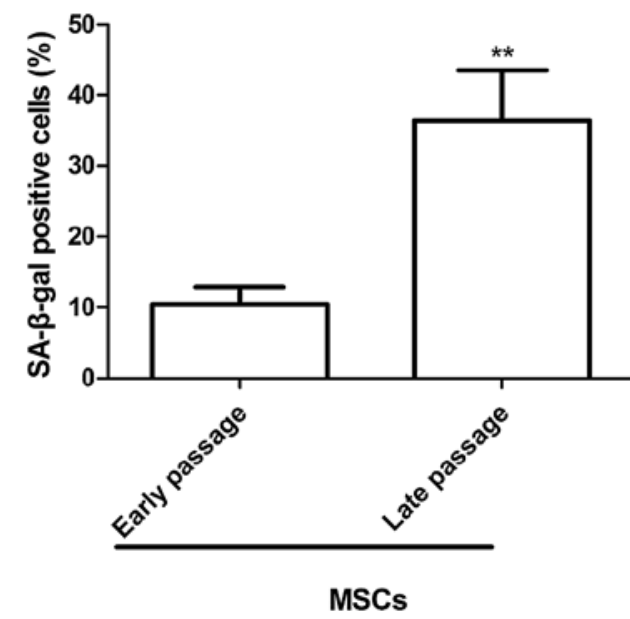

B

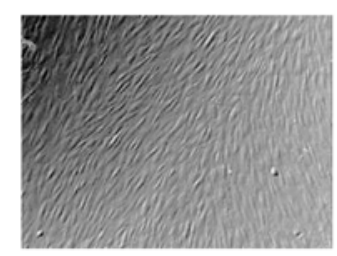

Passage 3

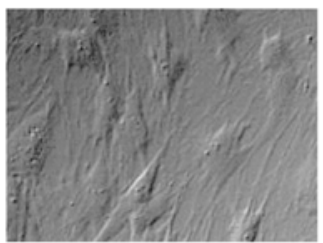

Passage 9

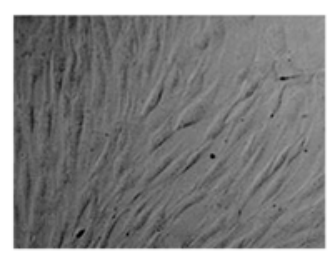

Passage 7

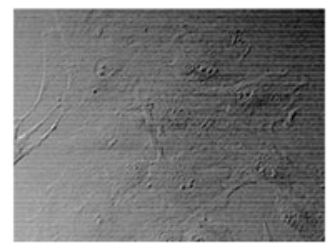

Passage 12

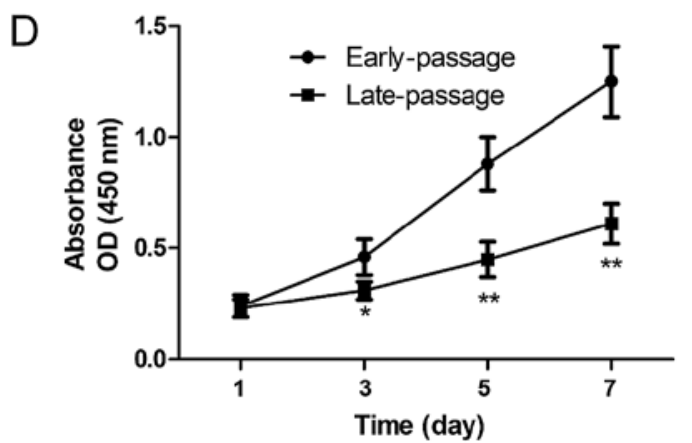

Figure 1. Characterization of mesenchymal stem cells (MSCs) at different passages. (A) Immunophenotypic characterization of MSCs by flow cytometry. The results indicated that the MSCs were positive for CD29, CD71 and CD90 but negative for CD45. (B) Morphology of MSCs at passages 3, 7, 9 and 12. (C) Early- and late-passage MSCs were stained with senescence-associated $\beta$-galactosidase (SA- $\beta$-gal) and the percentage of SA- $\beta$-gal positive cells was counted. (D) A cell proliferation assay (CCK-8) was performed on the early- and late-passage MSCs. ${ }^{*} \mathrm{P}<0.05,{ }^{* * *} \mathrm{P}<0.01$ vs. early-passage MSCs.

1:500; Cat. no. 4000; all from Cell Signaling Technology - New England Biolabs, Hitchin, UK). Following 3 washes with TBST, the membranes were incubated with goat anti-mouse IgG $(1: 5,000 ;$ Cat. no. Sc-2005) or goat anti-rabbit $(1: 5,000$; Cat. no. Sc-2004; both from Santa Cruz Biotechnology, Inc.) horseradish peroxidase (HRP) diluted in blocking buffer for $1 \mathrm{~h}$. Antibody binding was visualized using an enhanced chemiluminescence (ECL) western blotting detection system (Amersham Biosciences, Piscataway, NJ, USA). GAPDH was used for the normalization of protein loading and protein expression was measured by quantifying the density of immunoblots adjusted to GAPDH using image analysis software 3.1 Image J (Bio-Rad Laboratories).

Cell cycle analysis. The cells $\left(1 \times 10^{5}\right.$ cells $/ 10-\mathrm{cm}$ dish in diameter) were harvested by trypsinization, fixed with a fluorescence-activated cell sorting (FACS) lysing solution (BD Biosciences) and permeabilized with a FACS permeabilization solution (BD Biosciences). After being washed with PBS, the cells were resuspended in staining solution [containing $200 \mu \mathrm{g} / \mathrm{ml} \mathrm{RNase} \mathrm{A}$ and $20 \mu \mathrm{g} / \mathrm{ml}$ propidium iodide staining solution (Merck, Whitehouse Station, NJ, USA)], incubated for $30 \mathrm{~min}$, and analyzed for their DNA content using a FACScan flow cytometer (BD Biosciences).

Apoptosis assay. The detection of apoptotic cells was performed on cytospin preparations using the TUNEL assay according to the manufacturer's instructions (In Situ Cell Death Detection kit, AP; Roche Molecular Biochemicals, Mannheim, Germany) after the MSCs at passage 3 were treated with the indicated plasmid. The number of apoptotic cells was counted under a IX51 inverted microscope (Olympus Corp.) and averaged from 3 visual fields.

Treatment with LY294002. LY294002, a PI3K inhibitor, was obtained from Sigma-Aldrich. MSCs at passage 9 (late passage; $5 \times 10^{4}$ cells/well) were seeded into each well of a 24-well plate. Subsequently, 0.6 $\mu \mathrm{M}$ LY294002 was added and the cells were cultured for $48 \mathrm{~h}$ in DMEM (Invitrogen) supplemented with FBS at $37^{\circ} \mathrm{C}$ in a humidified atmosphere containing $5 \% \mathrm{CO}_{2}$. $\mathrm{PI} 3 \mathrm{~K}, \mathrm{p}-\mathrm{PI} 3 \mathrm{~K}, \mathrm{AKT}$ and $\mathrm{p}-\mathrm{AKT}$ protein expression was then determined by western blot analysis. 
A

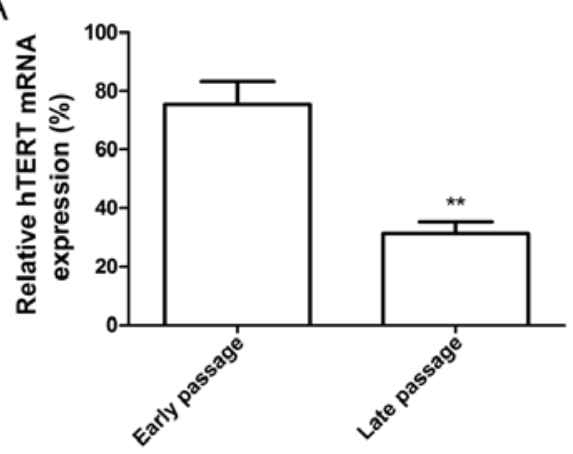

$\mathrm{C}$

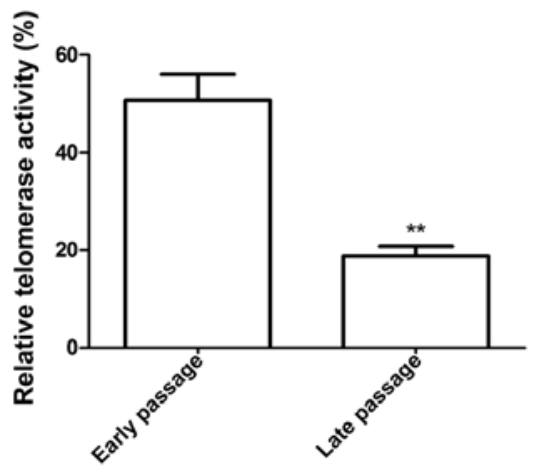

B
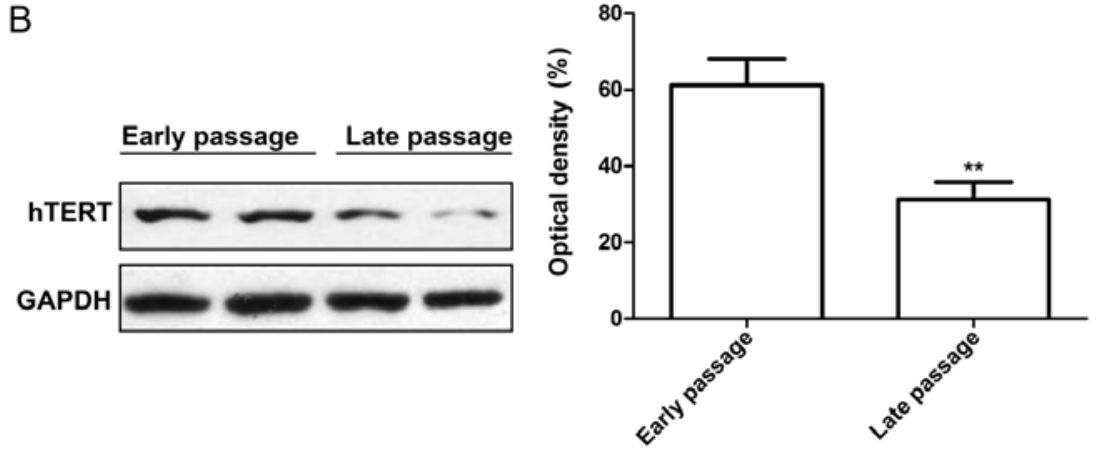

D

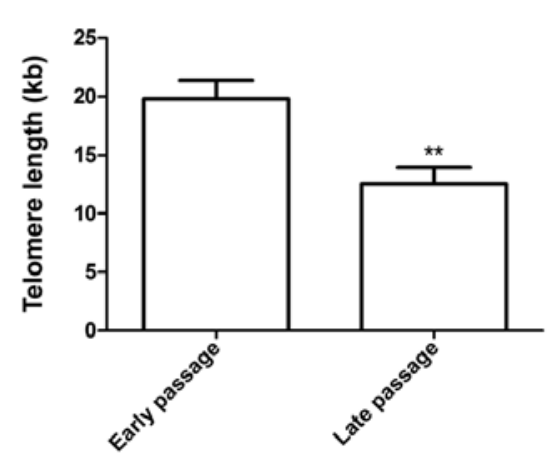

Figure 2. Human telomerase reverse transcriptase (hTERT) expression and telomerase activity and telomere length in early- and late-passage mesenchymal stem cells (MSCs). (A) mRNA expression levels and (B) protein expression levels were determined in early- and late-passage MSCs by RT-qPCR and western blot analysis, respectively. (C) Telomerase activity and (D) telomere length in early- and late-passage MSCs were measured. ${ }^{*} \mathrm{P}<0.05{ }^{* * *} \mathrm{P}<0.01$ vs. early-passage $\mathrm{MSCs}$.

Statistical analysis. Data are presented as the means \pm standard deviation (SD). Comparisons between 2 groups were made using the Student's t-test. Statistical differences among more than 2 groups were assessed by one-way analysis of variance (ANOVA). GraphPad Prism software version 6.01 (GraphPad Software, San Diego, CA, USA) was used for statistical analyses. A P-value $<0.05$ was considered to indicate a statistically significant difference.

\section{Results}

Characterization of MSCs at different passages. High-purity MSCs were isolated and obtained by density gradient centrifugation, adherence selection and the monoclonal culture system. The immunophenotype of the MSCs obtained from the Sprague-Dawley rats was assessed by flow cytometry using specific cell surface antigens. The MSCs were positive for the expression of the MSCs markers CD29, CD71 and CD90 but negative for CD45 expression (Fig. 1A). In addition, the morphological characteristics of the MSCs at different passages were observed under an inverted microscope. As shown in Fig. 1B, at passage 3, the cells had a whirlpool or fish-like shape. At passage 7, the MSCs became larger in size with an enlarged rough endoplasmic reticulum, microvilli desquamate, with many secondary lysosomes, suggesting that the MSCs entered senescence. At passage 9, large parts of the microvilli disappeared and the enlargement of the rough endoplasmic reticulum was more obvious. At passage 12, the MSCs had lost their normal shape, and karyopyknosis and heterochromatin were observed. To ascertain alterations in the senescence of the MSCs at different passages, SA- $\beta$-gal activity was analyzed. Compared with the early-passage MSCs (passage 3), the percentage of SA- $\beta$-galpositive cells and the staining intensity increased significantly in the late-passage MSCs (passage 9; Fig. 1C). In addition, we assessed the proliferation of MSCs at different passages by CCK-8 assay. We found that the proliferation of the late-passage MSCs decreased significantly compared with that of the earlypassage MSCs (Fig. 1D). These data indicate an increase in the senescene of MSCs at a late passage.

Senescence-associated alterations in hTERT expression, and in telomerase activity and telomere length in MSCs. To determine whether hTERT expression is altered in MSCs at different passages, we measured the hTERT expression levels by RT-qPCR and western blot analysis. Our results revealed that not only was the mRNA expression level of hTERT in the late-passage MSCs lower than that in the early-passage MSCs (Fig. 2A), but the protein expression level of hTERT was also significantly decreased in the late-passage MSCs compared to the early-passage MSCs (Fig. 2B). In addition, we evaluated telomerase activity and telomere length in the early- and latepassage MSCs. We discovered that telomerase activity (Fig. 2C) and telomere length (Fig. 2D) were significantly decreased in the late-passage MSCs compared with the early-passage MSCs.

hTERT modulates telomere length and telomerase activity in MSCs. It has been demonstrated that telomere attrition triggers telomere dysfunction, induces DNA damage and, consequently, cellular senescence (29). We wished to determine whether hTERT expression regulates telomerase activity. For this purpose, the MSCs at passage 9 were transfected with the plasmids, pSi-hTERT (for the downregulation of hTERT), and 

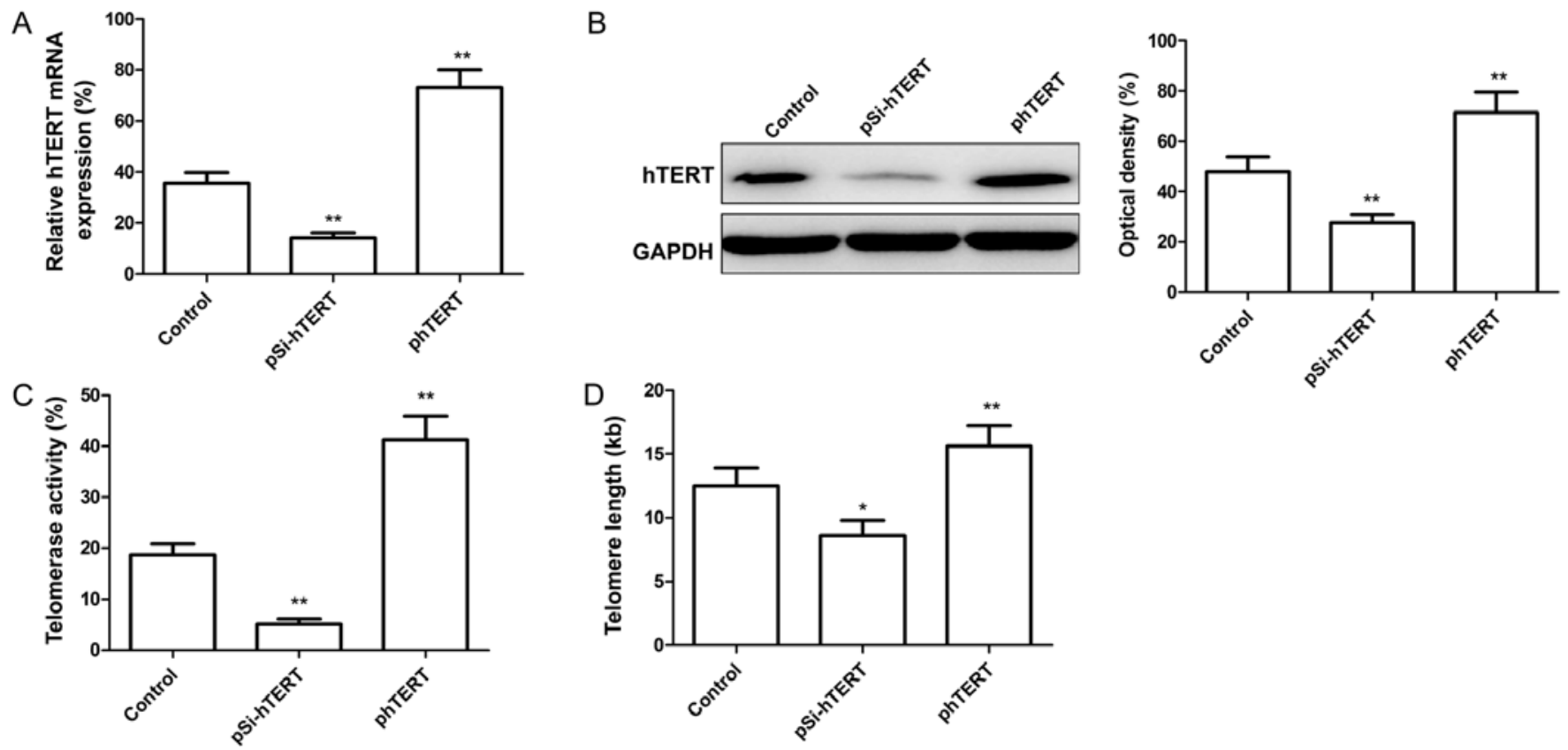

Figure 3. Human telomerase reverse transcriptase (hTERT) modulates telomerase length and telomere activity in mesenchymal stem cells (MSCs). (A) RT-qPCR analysis of hTERT mRNA levels and (B) western blot analysis of hTERT protein levels in MSCs at passage 9 following transfection with the plasmids, pSi-hTERT or phTERT. (C) Telomerase activity and (D) telomere length were determined in the MSCs at passage 9 following transfection with the plasmids, pSi-hTERT or phTERT. ${ }^{*} \mathrm{P}<0.05,{ }^{* * *} \mathrm{P}<0.01$ vs. control (untransfected cells were used as controls).

A

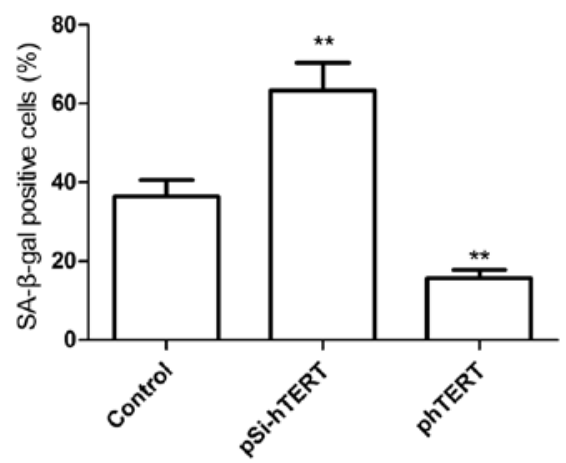

C

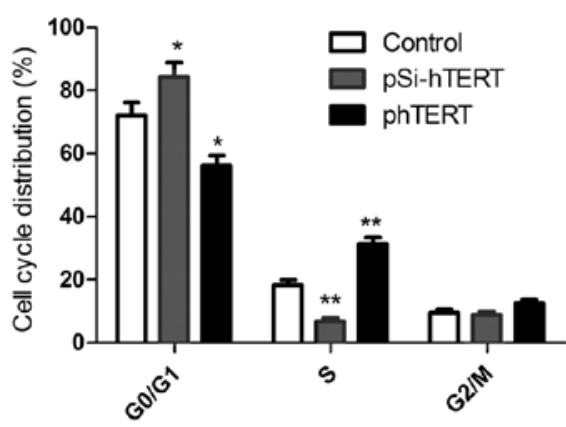

B
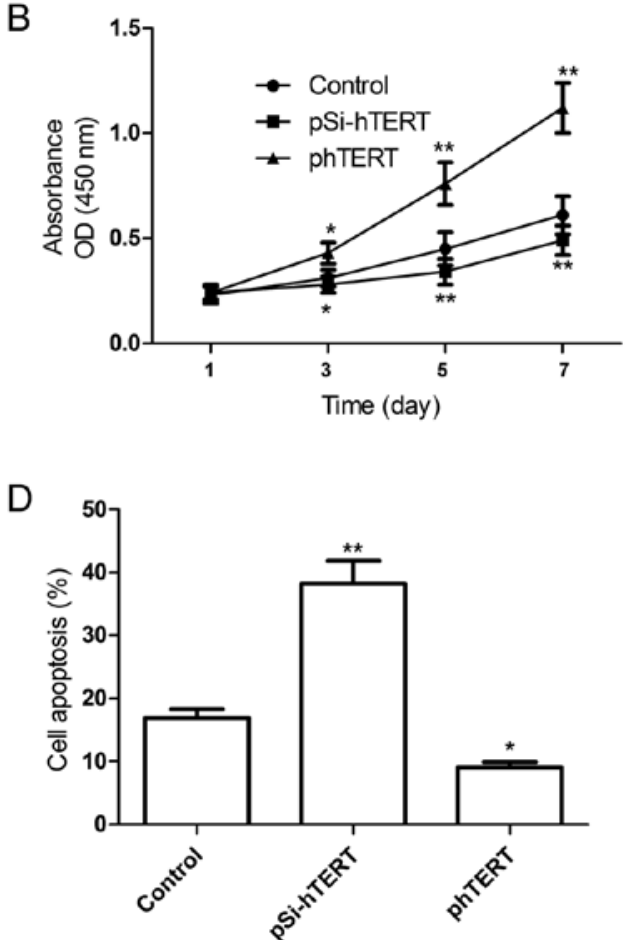

Figure 4. Human telomerase reverse transcriptase (hTERT) modulates the senescence, proliferation, cell cycle and the apoptosis of mesenchymal stem cells (MSCs) (A) Senescence, (B) cell proliferation, (C) cell cycle and (D) apoptosis were determined in the MSCs at passage 9 following transfection with the plasmids, pSihTERT or phTERT. ${ }^{*} \mathrm{P}<0.05,{ }^{* *} \mathrm{P}<0.01$ vs. control (untransfected cells were used as controls).

phTERT (for the upregulation of hTERT). The hTERT mRNA and protein expression levels were then measured by RT-qPCR and western blot analysis, respectively. Our results revealed that the hTERT mRNA and protein expression levels decreased significantly following transfection with pSi-hTERT (Fig. 3A and $\mathrm{B}$ ), whereas these levels significantly increased folloiwng transfection with phTERT (Fig. 3A and B). In addition, telomerase activity and telomere length in the MSCs were determined. We found that the downregulation of hTERT significantly reduced telomerase activity and telomere length in 
A
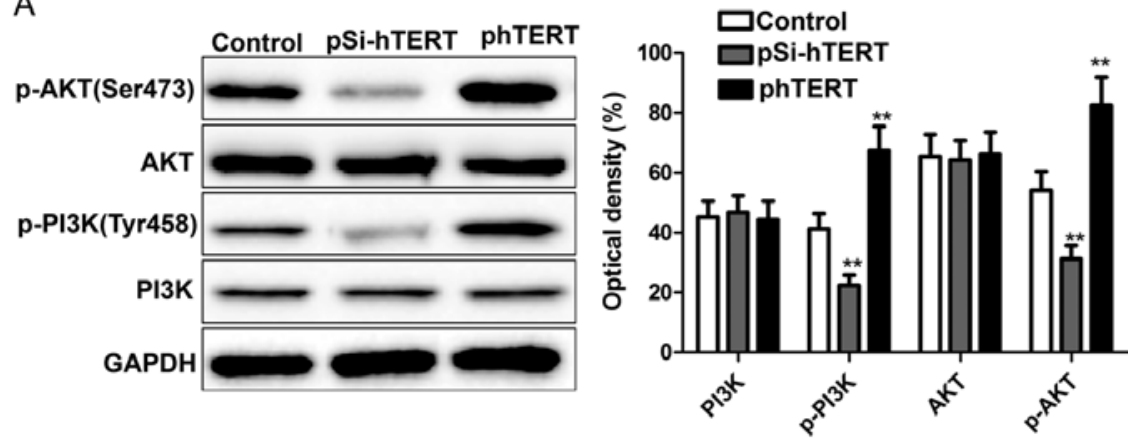

B

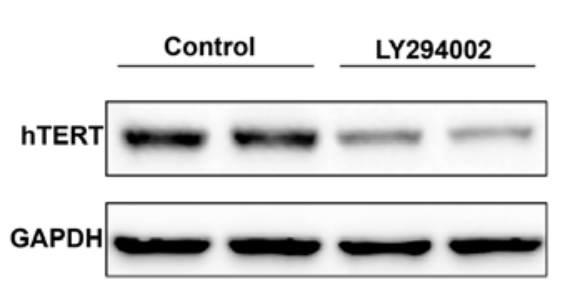

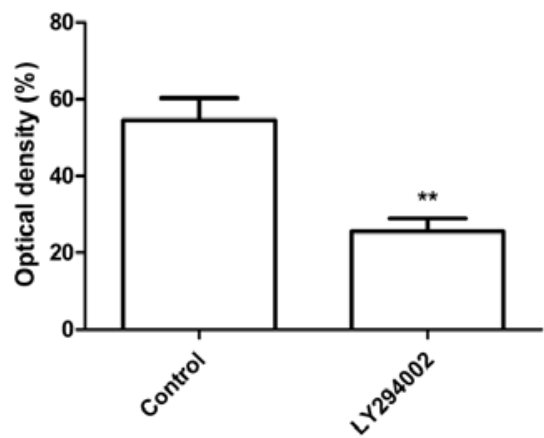

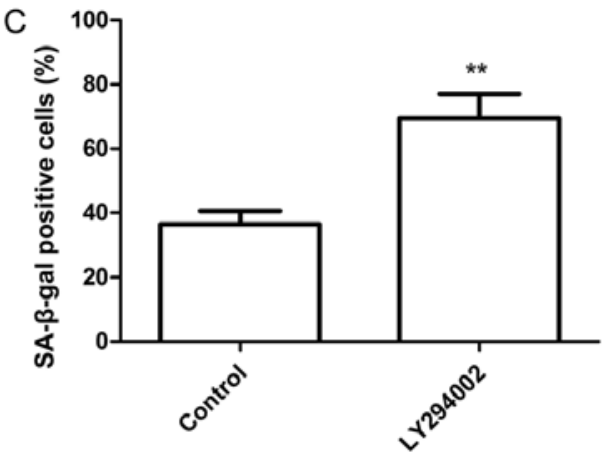

D

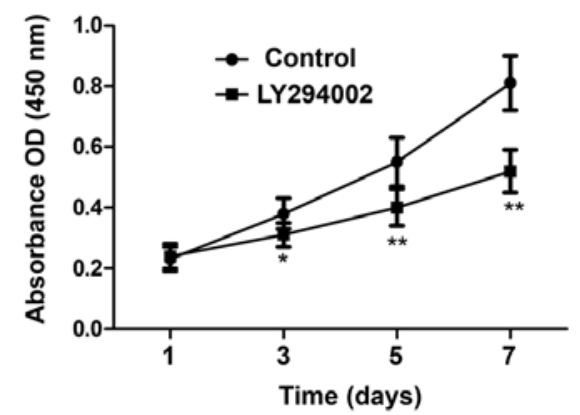

Figure 5. Human telomerase reverse transcriptase (hTERT) modulates the activatoin of the PI3K/AKT signal pathway in mesenchymal stem cells (MSCs). (A) PI3K, p-PI3K, AKT and p-AKT protein expression levels were determined in MSCs at passage 9 following transfection with the plasmids, pSi-hTERT or phTERT. (B) hTERT protein expression was determined in MSCs following treatment with LY294002. (C) Senescence and (D) proliferation of MSCs were determined following treatment with LY294002. ${ }^{*} \mathrm{P}<0.05,{ }^{* *} \mathrm{P}<0.01$ vs. control (untransfected cells were used as controls).

the MSCs (Fig. 3C and D), whereas the upregulation of hTERT significantly increased telomerase activity and telomere length in the MSCs (Fig. 3C and D).

hTERT modulates the senescence, proliferation, cell cycle and apoptosis of MSCs. To further determine the role of hTERT in regulating the senescence, proliferation, cell cycle and apoptosis of MSCs, the plasmids, pSi-hTERT or phTERT, were transfected into the MSCs at passage 9 (late passage), and the senescence, proliferation, cell cycle and apoptosis of the MSCs were then determined. Compared with the control group, the downregulation of hTERT induced cellular senescence (Fig. 4A; shown by an increase in the number of SA- $\beta$-gal-positive cells), decreased cell proliferation (Fig. 4B) and the percentage of cells in the $\mathrm{S}$ phase (Fig. 4C) and increased the percentage of apoptotic MSCs (Fig. 4D). However, the upregulation of hTERT significantly decreased the number of senescent cells (Fig. 4A), increased cell proliferation (Fig. 4B) and the percentage of cells in the S phase (Fig. 4C) and decreased the percentage of apoptotic late-passage MSCs (Fig. 4D). Taken together, these results suggest that hTERT overexpression prevents the replicative senescence of MSCs.

hTERT modulates the activation of the PI3K/AKT signaling pathway in MSCs. It has been demonstrated that the PI3K/ AKT pathway plays a role in stem cell self-renewal, maintenance and differentiation (30). In a recent study of ours, we demonstrated that the downregulation of hTERT inhibited the activation of the PI3K/AKT pathway (28). In the present study, we wished to determine whether hTERT modulates the activa- tion of the PI3K/AKT pathway in MSCs. At $24 \mathrm{~h}$ following transfection with pSi-hTERT or phTERT, the expression levels of PI3K, p-PI3K, AKT and p-AKT were measured by western blot analysis. We found that the downregulation of hTERT significantly decreased $\mathrm{p}$-PI3K and $\mathrm{p}$-AKT expression in the MSCs, whereas the upregulation of hTERT significantly increased $\mathrm{p}$-PI3K and p-AKT expression in the MSCs; no changes were observed in the levels of total PI3K and AKT in either group (Fig. 5A). To further determine whether the actiation of the PI3K/AKT signaling pathway is associated with the hTERT expression level in MSCs, the MSCs were treated with LY294002 (Sigma-Aldrich), a PI3K inhibitor, and the hTERT expression levels were then measured by western blot analysis. The results revealed that LY294002 significantly inhibited hTERT expression (Fig. 5B). In addition, the proliferation and senescence of the MSCs were determined following treatment with LY294002. The results revealed that treatment with LY294002 led to a significant increase in cellular senescence (Fig. 5C; as shown by an increase in the number of SA- $\beta$-gal-positive cells) and a decreased in the proliferation of MSCs (Fig. 5D). These findings suggest that hTERT mediates the senescence of MSCs through the PI3K/ AKT signaling pathway.

\section{Discussion}

MSCs are one of the most promising resources for cell and gene therapy for osteogenesis imperfecta, the tissue engineering of cartilage and bone and post-transplant immune reconstitution due to their versatile plasticity in vitro and in vivo (32). 
However, their clinical application and basic research is limited as primary MSCs have a limited lifespan. It is crucial to understand the mechanisms regulating cellular senescence, so that ways can be found to extend the lifespan of MSCs.

Cellular senescence is a complex process that, thus far, remains largely unknown. Previous studies have demonstrated that telomere attrition triggers telomere dysfunction, and induces DNA damage, leading to cellular senescence $(29,33)$. Telomere attrition may thus be closely related to impaired telomerase activity and telomere length as the course of the telomeric DNA elongation is dependent on telomerase catalysis and may results in a reduction in telomerase activity in MSC (34). It has previously been demonstrated that if telomere shortening is not balanced by elongation, it leads to cell death, cellular senescence or abnormal cell proliferation (35). In the present study, we found that the long-term in vitro culture of MSCs led to cellular senescence, and telomerase activity and telomere length were both decreased in the late-passage MSCs compared with the early-passage MSCs. These findings suggested that telomeres play a key role in the senescence of long-term cultured MSCs.

It has been demonstrated that the ectopic expression of hTERT, the catalytic component of telomerase, leads to telomere elongation and extends the lifespan of a number of cell types (11-13). The upregulation of hTERT in MSCs has been shown to enhance their stem-like properties without affecting their potential to differentiate into osteocytes, adipocytes and chondrocytes (20-25). Consistent with these results, in the present study, we found that the downregulation of hTERT by siRNA markedly decreased telomere length and telomerase activity in the MSCs, whereas the overexpression of hTERT increased telomere length and telomerase activity in the MSCs. The downregulation of hTERT led to a decrease in the proliferation and an increase in the number of senescent and apoptotic MSCs, whereas the upregulation of hTERT led to an increase in cell proliferation and a decrease in the number of senescent and apoptotic MSCs.

It was well known that AKT (also known as PKB), a serine/ threonine protein kinase, plays a central role in regulating cell survival, metabolism and protein synthesis through the phosphorylation of its numerous substrates (36). Previous studies have shown that AKT plays a role in the self-renewal, maintenance and differentiation of several types of stem cells, including pluripotent stem cells (31), neuronal stem cells (37) and epithelial stem cells (38). In addition, AKT is able to phosphorylate hTERT and activate telomerase activity (39). Two putative AKT phosphorylation sites within hTERT (serine residues at 227 and 824) have been identified. It has been reported that AKT enhances telomerase activity through the phosphorylation and nuclear translocation of hTERT $(40,41)$. In the present study, we found that the downregulation of hTERT significantly inhibited p-PI3K and p-AKT expression in the MSCs, whereas the upregulation of hTERT significantly increased p-PI3K and p-AKT expression in the MSCs. This pathway was blocked with LY294002 (PI3K inhibitor), which led to a decrease in hTERT expression, increase in cellular senescence and a decrease in the proliferation of MSCs. Our findings reveal a previously unknown regulatory mechanism of hTERT; namely that hTERT mediates the senescence of MSC through the PI3K/AKT signaling pathway.
In conclusion, in the present study, we demonstrated that hTERT expression, telomerase activity and telomere length were decreased in late-passage MSCs compared to earlypassage MSCs. The upregulation of hTERT in the late-passage MSCs prevented cellular senescence and apoptosis, increased cell proliferation, and increased telomerase activity and telomere length. We also demonstrated that the upregulation of hTERT activates the PI3K/AKT signaling pathway. and that the inhibition of the activation of the PI3K/AKT signaling pathway with LY294002 led to a decrease in hTERT expression, an increase in cellular senescence and a decrease in the proliferation of MSCs. These findings suggest that hTERT mediates the senescence of MSCs through the PI3K/AKT signaling pathway.

\section{Acknowledgements}

The present study was supported by the Scientific Research Project of Jilin Provincial Bureau of Health (2013ZC005; 2013Z028); the Jilin Provincial Science and Technology Projects (20130101130JC); the Norman Bethune Program of Jilin University (2012204); and The Project-sponsored by SRF for ROCS, SEM, and The Project-Basic Study by Jilin University.

\section{References}

1. Bayati V, Hashemitabar M, Gazor R, Nejatbakhsh R and Bijannejad D: Expression of surface markers and myogenic potential of rat bone marrow- and adipose-derived stem cells: a comparative study. Anat Cell Biol 46: 113-121, 2013.

2. Wakitani S, Saito T and Caplan AI: Myogenic cells derived from rat bone marrow mesenchymal stem cells exposed to 5-azacytidine. Muscle Nerve 18: 1417-1426, 1995.

3. Javazon EH, Colter DC, Schwarz EJ and Prockop DJ: Rat marrow stromal cells are more sensitive to plating density and expand more rapidly from single-cell-derived colonies than human marrow stromal cells. Stem Cells 19: 219-225, 2001.

4. Beyer Nardi N and da Silva Meirelles L: Mesenchymal stem cells: isolation, in vitro expansion and characterization. Handb Exp Pharmacol 174: 249-282, 2006.

5. Brighton CT and Hunt RM: Early histological and ultrastructural changes in medullary fracture callus. J Bone Joint Surg Am 73: 832-847, 1991.

6. Caplan AI: Mesenchymal stem cells. J Orthop Res 9: 641-650, 1991.

7. Li J and Pei M: Cell senescence: A challenge in cartilage engineering and regeneration. Tissue Eng Part B Rev 18: 270-287, 2012.

8. Allsopp RC, Vaziri H, Patterson C, Goldstein S, Younglai EV, Futcher AB, Greider CW and Harley CB: Telomere length predicts replicative capacity of human fibroblasts. Proc Natl Acad Sci USA 89: 10114-10118, 1992.

9. Broccoli D, Young JW and de Lange T: Telomerase activity in normal and malignant hematopoietic cells. Proc Natl Acad Sci USA 92: 9082-9086, 1995.

10. Counter CM, Avilion AA, LeFeuvre CE, Stewart NG, Greider CW, Harley CB and Bacchetti S: Telomere shortening associated with chromosome instability is arrested in immortal cells which express telomerase activity. EMBO J 11: 1921-1929, 1992.

11. Vaziri $\mathrm{H}$ and Benchimol S: Reconstitution of telomerase activity in normal human cells leads to elongation of telomeres and extended replicative life span. Curr Biol 8: 279-282, 1998.

12. Bodnar AG, Ouellette M, Frolkis M, Holt SE, Chiu CP, Morin GB, Harley CB, Shay JW, Lichtsteiner S and Wright WE: Extension of life-span by introduction of telomerase into normal human cells. Science 279: 349-352, 1998.

13. Greider CW and Blackburn EH: A telomeric sequence in the RNA of Tetrahymena telomerase required for telomere repeat synthesis. Nature 337: 331-337, 1989.

14. Feng J, Funk WD, Wang SS, Weinrich SL, Avilion AA, Chiu CP Adams RR, Chang E, Allsopp RC, Yu J, et al: The RNA component of human telomerase. Science 269: 1236-1241, 1995. 
15. Kassem M, Abdallah BM, Yu Z, Ditzel N and Burns JS: The use of hTERT-immortalized cells in tissue engineering. Cytotechnology 45: 39-46, 2004.

16. Tao Q, Lv B, Qiao B, Zheng CQ and Chen ZF: Immortalization of ameloblastoma cells via reactivation of telomerase function: Phenotypic and molecular characteristics. Oral Oncol 45: e239-e244, 2009.

17. Morales CP, Holt SE, Ouellette M, Kaur KJ, Yan Y, Wilson KS, White MA, Wright WE and Shay JW: Absence of cancer-associated changes in human fibroblasts immortalized with telomerase. Nat Genet 21: 115-118, 1999.

18. Rajamani K, Lin YC, Wen TC, Hsieh J, Subeq YM, Liu JW, Lin PC, Harn HJ, Lin SZ and Chiou TW: The antisenescence effect of trans-cinnamaldehyde on adipose-derived stem cells. Cell Transplant 24: 493-507, 2015.

19. Yang J, Chang E, Cherry AM, Bangs CD, Oei Y, Bodnar A, Bronstein A, Chiu CP and Herron GS: Human endothelial cell life extension by telomerase expression. J Biol Chem 274: 26141-26148, 1999.

20. Bischoff DS, Makhijani NS and Yamaguchi DT: Constitutive expression of human telomerase enhances the proliferation potential of human mesenchymal stem cells. Biores Open Access 1: 273-279, 2012.

21. Huang G, Zheng Q, Sun J, Guo C, Yang J, Chen R, Xu Y, Wang G, Shen D, Pan Z, et al: Stabilization of cellular properties and differentiation mutilpotential of human mesenchymal stem cells transduced with hTERT gene in a long-term culture. J Cell Biochem 103: 1256-1269, 2008.

22. Kobune M, Kawano Y, Ito Y, Chiba H, Nakamura K, Tsuda $H$ Sasaki K, Dehari H, Uchida H, Honmou O, et al: Telomerized human multipotent mesenchymal cells can differentiate into hematopoietic and cobblestone area-supporting cells. Exp Hematol 31: 715-722, 2003.

23. Piper SL, Wang M, Yamamoto A, Malek F, Luu A, Kuo AC and Kim HT: Inducible immortality in hTERT-human mesenchymal stem cells. J Orthop Res 30: 1879-1885, 2012.

24. Moffatt-Jauregui CE, Robinson B, de Moya AV, Brockman RD, Roman AV, Cash MN, Culp DJ and Lamont RJ: Establishment and characterization of a telomerase immortalized human gingival epithelial cell line. J Periodontal Res 48: 713-721, 2013.

25. Yang YX, Miao ZC, Zhang HJ, Wang Y, Gao JX and Feng MF: Establishment and characterization of a human telomerase catalytic subunit-transduced fetal bone marrow-derived osteoblastic cell line. Differentiation 75: 24-34, 2007.

26. Scutt A and Bertram P: Bone marrow cells are targets for the anabolic actions of prostaglandin E2 on bone: induction of a transition from nonadherent to adherent osteoblast precursors. J Bone Miner Res 10: 474-487, 1995.

27. Galderisi U, Helmbold H, Squillaro T, Alessio N, Komm N, Khadang B, Cipollaro M, Bohn W and Giordano A: In vitro senescence of rat mesenchymal stem cells is accompanied by downregulation of stemness-related and DNA damage repair genes. Stem Cells Dev 18: 1033-1042, 2009.
28. Shi YA, Zhao Q, Zhang LH, Du W, Wang XY, He X, Wu S and Li YL: Knockdown of hTERT by siRNA inhibits cervical cancer cell growth in vitro and in vivo. Int J Oncol 45: 1216-1224, 2014.

29. Palm W and de Lange T: How shelterin protects mammalian telomeres. Annu Rev Genet 42: 301-334, 2008.

30. Follo MY, Manzoli L, Poli A, McCubrey JA and Cocco L: PLC and PI3K/Akt/mTOR signalling in disease and cancer. Adv Biol Regul 57: 10-16, 2015.

31. Yoon KA, Cho HS, Shin HI and Cho JY: Differential regulation of CXCL5 by FGF2 in osteoblastic and endothelial niche cells supports hematopoietic stem cell migration. Stem Cells Dev 21: 3391-3402, 2012.

32. Pittenger MF, Mackay AM, Beck SC, Jaiswal RK, Douglas R, Mosca JD, Moorman MA, Simonetti DW, Craig S and Marshak DR: Multilineage potential of adult human mesenchymal stem cells. Science 284: 143-147, 1999.

33. Stewart JA, Chaiken MF, Wang F and Price CM: Maintaining the end: Roles of telomere proteins in end-protection, telomere replication and length regulation. Mutat Res 730: 12-19, 2012.

34. Serakinci N, Graakjaer J and Kolvraa S: Telomere stability and telomerase in mesenchymal stem cells. Biochimie 90: 33-40, 2008.

35. Fajkus J, Simícková M and Maláska J: Tiptoeing to chromosome tips: facts, promises and perils of today's human telomere biology. Philos Trans R Soc Lond B Biol Sci 357: 545-562, 2002.

36. Dimitrova V and Arcaro A: Targeting the PI3K/AKT/mTOR signaling pathway in medulloblastoma. Curr Mol Med 15: 82-93, 2015.

37. Chell JM and Brand AH: Nutrition-responsive glia control exit of neural stem cells from quiescence. Cell 143: 1161-1173, 2010.

38. Sewell GW, Marks DJ and Segal AW: The immunopathogenesis of Crohn's disease: a three-stage model. Curr Opin Immunol 21: 506-513, 2009.

39. Kang SS, Kwon T, Kwon DY and Do SI: Akt protein kinase enhances human telomerase activity through phosphorylation of telomerase reverse transcriptase subunit. J Biol Chem 274: 13085-13090, 1999.

40. Kawagoe J, Ohmichi M, Takahashi T, Ohshima C, Mabuchi S, Takahashi K, Igarashi H, Mori-Abe A, Saitoh M, Du B, et al: Raloxifene inhibits estrogen-induced up-regulation of telomerase activity in a human breast cancer cell line. J Biol Chem 278: 43363-43372, 2003.

41. Kimura A, Ohmichi M, Kawagoe J, Kyo S, Mabuchi S, Takahashi T, Ohshima C, Arimoto-Ishida E, Nishio Y, Inoue M, et al: Induction of hTERT expression and phosphorylation by estrogen via Akt cascade in human ovarian cancer cell lines. Oncogene 23: 4505-4515, 2004. 